\title{
Psychosocial Determinants of Self-care Behavior in Outpatients with Heart Failure: A Test of Pender s Health Promotion Model
}

\author{
Rasoul Heshmati1 ${ }^{1,}$, Mahasti Alizadeh ${ }^{2}$, Solmaz Javadpour ${ }^{3}$ and Nilufar Alizada ${ }^{4}$ \\ 1 Social Determinants of Health Research Center, Health Management and Safety Promotion Research Institute, Tabriz University of Medical Sciences, Tabriz, Iran \\ 2 Social Determinants of Health Research Center, Health Management and Safety Promotion Research Institute, Tabriz University of Medical Sciences, Tabriz, Iran \\ 3 Department of Psychology, Faculty of Humanities, Marand Branch, Islamic Azad University, Marand, Iran \\ 4 Department of Psychology, School of Humanities, Education and Social Sciences, Khazar University, Baku, Azerbaijan
}

* Corresponding author: Rasoul Heshmati, Department of Psychology, Faculty of Education and Psychology, University of Tabriz, Tabriz, Iran. Email: psy.heshmati@gmail.com

Received 2020 October 19; Revised 2020 November 03; Accepted 2020 November 12.

\begin{abstract}
Background: Patients with heart failure need to be engaged in adequate cardiac self-care behaviors helping to prevent the development of the disease and ameliorate their health status. However, the conceptual model of the present study has not been tested in previous studies among patients with heart failure.

Objectives: The present study aimed to investigate the psychosocial determinants based on Pender's health promotion model (HPM) affecting self-care behavior among outpatients suffering from heart failure.

Methods: In this cross-sectional study, a total of 200 patients suffering from heart failure were selected from the outpatient clinics of Tabriz, Iran, using convenience sampling and assessed for self-self-care behaviors and major concepts of HPM via self-administered questionnaires. Path analysis was used in order to analyze the conceptual model.

Results: The present hypothetical model showed a good fit. Perceived benefits and activity-related affect directly affected self-care behaviors. Bootstrapping mediation analyses indicated that perceived self-efficacy, perceived barriers, perceived benefits, and activityrelated affect indirectly affected self-care behaviors through commitment to action.

Conclusion: The commitment to action can help to promote self-care behaviors among the subjects suffering from heart failure. The interventions should take the role of predictive variables of this study and commitment to action into account in these patients.

Keywords: Heart failure, Health promotion, Psychosocial factors, Self-care behaviors
\end{abstract}

\section{Background}

The leading causes of mortality and disability in Iran are considered cardiovascular diseases (1). The main causes of this disease are problems in self-care and unhealthy behaviors (2). Heart failure is a chronic cardiovascular disease and one of the leading causes of mortality and burden in low- and middleincome countries (3). The prevalence rates of heart failure are within the ranges of $0.4-4.3 \%$ and $2-20 \%$ in the general population and aging population of over 75 years, respectively (4), which is estimated to increase by two times in the next two decades (5-6). The 1-year heart failure mortality rate was reported as $32 \%$ in Iran falling within the range of this rate in other countries (7).

One of the main criteria in the improvement of treatment efficacy is self-care behavior in these patients. Self-care activities can lead an individual to maintain health and well-being, improve adaptability, and decrease the disability of patients and treatment cost of diseases (8). In addition, self-care behaviors reduce the severe symptoms of diseases, improve clinical outcomes, and decrease the rate of hospitalization (9). However, self-care among patients suffering from heart failure in Iran was not satisfactory and needs to be improved(10).
The health promotion model (HPM) was proposed by Pender to alter self-care behaviors and improve health (11). The HPM is based on social cognitive theory in which cognitive-perceptual determinants (i.e., perceived benefits, barriers, and self-efficacy) affect engagement in health-promoting behaviors. Modifying factors are considered to interrelate with each other to affect cognitive-perceptual processes (12). This model has the variables comprising the main part of the treatment. These variables provide a rich source of interventional content and strategies $(13,14)$.

Based on the evidence, it was shown that perceived self-efficacy was the main determinant in health-promoting behaviors, and perceived religious support had direct and indirect effects on healthpromoting behaviors via influencing perceived selfefficacy, barriers, and benefits (15). Understanding the perceived barriers to health-promoting behaviors and removing them lead to the enhancement of the quality of patient care and method for planning the training services for patients to change their behaviors $(16,17)$. According to the literature, cardiovascular patients with a better perception of the benefits of compliance had low levels of perceived barriers to health-promoting behaviors (18). In addition, the relationship between self-efficacy and 
health-promoting behaviors was shown in diabetic and hypertension patients $(19,20)$.

\section{Objectives}

Although HPM has been widely used for different diseases, still the role of this model in promoting health, especially self-care behaviors in outpatients suffering from heart failure, has been poorly investigated. Furthermore, the determinants of selfcare behaviors based on Pender's HPM have not been examined as a structural model in Iranian patients with heart failure in previous studies. Therefore, the current study aimed to examine the effects of Pender s HPM components on the self-care behaviors of outpatients suffering from heart failure.

\section{Methods}

\subsection{Participants and procedure}

In this cross-sectional study, a total of 200 individuals suffering from heart failure were selected from the outpatient clinics of Tabriz, Iran, using convenience sampling within October and September 2017. In this study, $60 \%$ and $40 \%$ of the participants were female and male, respectively. The patients were reported within the age range of 61-83 years. The inclusion criteria were diagnosis of heart failure, age of over 60 years, and willingness to participate in the study. The exclusion criteria were comorbidity with other chronic diseases and history of psychological disorders.

After accepting to participate, in case of noncompliance with the inclusion and exclusion criteria, the individuals were excluded from the rest of the study, and only those patients were selected recently undergoing medical emergency with relative improvement and willingness to answer questions. Ethical approval was acquired from the Research Ethics Committee of Tabriz University of Medical Sciences, Tabriz, Iran. The study subjects were explained about the objectives of the study and reassured of the confidentiality of their information. All the participants were notified that they were free to withdraw from the study at any time. The questionnaires were not signed, and none of the participants answers were discussed in this study.

\subsection{Measures}

3.2. 1. European Heart Failure Self-Care Behavior Scale

The European Heart Failure Self-Care Behavior Scale was developed by Jaarsma et al. (12). This scale consists of 12 items that are rated based on a 5-point Likert from entirely true (no. 1) to entirely false (no. 5 ). The score range is considered within 12-16, in which the higher scores show lower self-care behaviors. The psychometric property of the scale was implemented in some Iranian studies $(21,22)$, and the Cronbach s alpha was equal to 0.71 (21). The internal consistency of the scale for the current study was reported as 0.88 .

\subsection{Perceived self-efficacy}

This scale was derived from a scale developed by Smith et al. with 10 items in eight parts, including the achievement of health, no achievement of optimal health, difficulty in finding the solution, success in health plans, achievement of goals, attempts for the achievement of health, belief that plans do not work, and belief that plans work. The answers are based on a 5-point Likert scale varying from "strongly agree" to "strongly disagree". The overall score of the scale is within the range of 8-14. As the acquired score increases, the capacity of the respondents for controlling the consequences and effects of their health-associated plans increases (23). The psychometric properties of this scale have been confirmed in a study by Smith et al. (23). In the current study, the internal consistency of the scale was excellent $(\alpha=0.91)$.

\subsection{Perceived barrier}

This scale was adapted from a questionnaire developed by Becker et al. (24) with 18 items in six parts, including a lack of facilities, support, information, and time, physical problems, and disinterestedness. The items of the scale are scored based on a 4-point Likert scale from "never" to "always". The overall scores are within the range of 18-72. Higher scores show more barriers to perform self-care behaviors (24). This scale has demonstrated excellent psychometric properties, including high concurrent validity, with other measures of perceived barriers (24). In addition, in the present study, this scale had an appropriate internal consistency $(\alpha=0.78)$.

\subsection{Perceived benefits}

The scale of perceived benefits was developed by Mohammadian et al. with 20 items rated according to a 7-point Likert scale (completely false: 1 point to completely true: 7 points). This tool evaluates the anticipated positive outcomes that will occur resulting from health-promoting behaviors. The score of the scale is within the range of 20-140. Higher scores indicate more perceived benefits for healthpromoting behaviors (25). The internal consistency of this scale reported by Mohammadian et al. was good (25). The internal consistency of the scale for the current study was reported as 0.79 .

\subsection{Perceived affect}

This scale was derived from a questionnaire developed by Watson et al. (26) with 20 items in six parts, including creative, enthusiastic, interested, nervous, guilty, and strong. This tool assesses perceived positive or negative emotions occurring before, during, and after the behavior. The items are scored based on a 5-point Likert scale from "not at 
all" to "absolutely". The total score of the scale is within the range of 20-100. In a study carried out by Watson et al. (26), this scale had an appropriate validity and internal consistency. In the current study, the perceived effect scale had adequate internal consistency $(\alpha=0.75)$.

\subsection{Perceived social support}

The perceived social support scale was adopted from a study conducted by Canty-Mitchell and Zimet (27) with 12 items in three parts, including family support, friend support, and particular individual support. This scale assesses cognition concerning the behaviors, beliefs, or attitudes of others. Therefore, it especially measures interpersonal influences. The items are scored based on a 5-point Likert scale from "strongly agree" to "strongly disagree". The overall score of the scale is within the range of 12-16. Higher scores indicate receiving higher support from friends, family, and other important individuals (27). The psychometric properties of the perceived social support scale are well established (20). In the present study, the internal consistency of the scale was high $(\alpha=0.91)$.

\subsection{Situational influences}

For the evaluation of situational influences, a questionnaire was designed based on Pender's HPM with 10 items. This instrument assesses the personal perceptions of any given situation that can boost or prevent self-care behaviors. The items are scored based on a 4-point Likert scale from strongly agree (5) to strongly disagree (1). The overall scores are within the range of 10-50. Higher scores indicate higher levels of situational influences. In the present study, Cronbach s alpha for this questionnaire was reported as 0.86 .

\subsection{Commitment to action}

For the evaluation of commitment to action, an assessment tool was developed based on Pender s theory evaluating the intention and identification of a planned strategy leading to display health-promoting behaviors. This assessment tool consists of 10 items. This instrument is rated based on a 5-point Likert scale from strongly agree (5) to strongly disagree (1). The scores obtained for each item are within the range of 10-50. Higher scores indicate increased reported commitment to action. In the present study, Cronbach s alpha for this tool was reported as 0.77 .

\subsection{Statistical analysis}

The data were analyzed using SPSS software (version 22) and Amos software (version 18). Firstly, questionnaire data were considered for missing item responses. Totally, $0.005 \%$ of the items were missing from the questionnaire data. Therefore, a single imputation applying the expectation-maximization algorithm was used to replace these missing items. Pearson correlation coefficient was applied to investigate the relationships between the variables. The path analysis was performed to determine whether the commitment to action mediated the relationship between the predictive variables and self-care behaviors.

In the hypothesized mediation model, perceived self-efficacy, perceived barriers, perceived benefits, activity-related affect, situational influences, and interpersonal impacts were the independent variables. Moreover, commitment to action was the mediator variable, and self-care behavior was the dependent variable. The bootstrapping method was used for mediation analysis. A mediation effect was noticed to be significant at a level of 0.05 if biascorrected 95\% confidence interval (CI) of 2,000 bootstrap samples did not include 0 .

For the evaluation of the goodness-of-fit of the present models, the current study reported on the goodness of fit index (GFI), adjusted goodness of fit index (AGFI), incremental fit index (IFI), Tucker-Lewis index (TLI), comparative fit index criterion (CFI), and root mean square error of approximation (RMSEA). For the GFI, AGFI, IFI, TLI, and CFI, the values of $\geq 0.90$ were considered indicative of a good fit. An RMSEA value of $\leq 0.08$ was considered adequate.

\section{Results}

\subsection{Correlational analysis}

Table 1 tabulates the correlational analysis of the

\begin{tabular}{|c|c|c|c|c|c|c|c|c|}
\hline Variable & 1 & 2 & 3 & 4 & 5 & 6 & 7 & 8 \\
\hline Self-care behaviors & 1 & & & & & & & \\
\hline Commitment to action & $0.60^{* *}$ & 1 & & & & & & \\
\hline Perceived self-efficacy & $0.46^{* *}$ & $0.42^{* *}$ & 1 & & & & & \\
\hline Perceived barriers & $-0.40^{* *}$ & $-0.36^{* *}$ & $-0.38^{* *}$ & 1 & & & & \\
\hline Perceived benefits & $0.45^{* *}$ & $0.38^{* *}$ & $0.42^{* *}$ & $-0.33^{* *}$ & 1 & & & \\
\hline Activity-related affect & $0.48^{* *}$ & $0.35^{* *}$ & $0.48^{* *}$ & $-0.28^{* *}$ & $0.22^{* *}$ & 1 & & \\
\hline Situational influences & $0.38^{* *}$ & $0.28^{* *}$ & $0.31^{* *}$ & $-0.38^{* *}$ & $0.29^{* *}$ & $0.43^{* *}$ & 1 & \\
\hline Interpersonal influences & $0.45^{* *}$ & $0.36^{* *}$ & $0.52^{* *}$ & $-0.55^{* *}$ & $0.48^{* *}$ & $0.45^{* *}$ & $0.26^{* *}$ & 1 \\
\hline Mean & 41.0 & 22.48 & 39.0 & 34.0 & 55.0 & 10.0 & 9.0 & 60.0 \\
\hline SD & 22.18 & 10.02 & 15.34 & 13.88 & 23.11 & 3.05 & 2.12 & 27.14 \\
\hline
\end{tabular}

SD: Standard deviation

${ }^{* *}$ Correlation significance at 0.01 (2-tailed)

*Correlation significance at 0.05 (2-tailed) 
variables. It was shown that perceived self-efficacy ( $\mathrm{r}=0.46 ; \mathrm{P}<0.01)$, perceived benefits $(\mathrm{r}=0.45 ; \mathrm{P}<0.01)$, activity-related affect $(\mathrm{r}=0.48 ; \mathrm{P}<0.01)$, situational influences $(\mathrm{r}=0.38 ; \mathrm{P}<0.01)$ and interpersonal impacts $(r=0.45 ; \mathrm{P}<0.01)$ had positive relations with self-care behaviors. Therefore, self-care behaviors increased with an elevation of perceived self-efficacy, perceived benefits, activity-related affect, situational influences, and interpersonal impacts. Conversely, perceived barriers had a negative relationship with self-care behaviors $(\mathrm{r}=-0.40 ; \mathrm{P}<0.01)$, indicating that self-care behaviors decreased with an elevation of perceived barriers. In addition, commitment to action positively correlated with self-care behaviors $(\mathrm{r}=0.60 ; \mathrm{P}<0.01)$.

\subsection{Model fitness}

Table 2 shows fit indices in the present study. According to the obtained results, the modified model had sufficient goodness-of-fit (GFI=0.90; AGFI=0.86; $\mathrm{IFI}=0.91 ; \mathrm{TLI}=0.90 ; \mathrm{CFI}=0.92 ; \mathrm{RMSEA}=0.07$ ).

\subsection{Path coefficients}

Figure 1 illustrates the path coefficients of the variables. Perceived self-efficacy significantly predicted commitment to action $(\beta=0.19 ; \mathrm{P}<0.05)$. In addition, the association between perceived barriers and commitment to action was significant $(\beta=-0.17$; $\mathrm{P}<0.05)$. The aforementioned associations were in the predicted direction. As hypothesized, the pathway from perceived benefits to commitment to action was significant $(\beta=0.21 ; \mathrm{P}<0.01)$. Activityrelated affect significantly predicted commitment to action $(\beta=0.16 ; \mathrm{P}<0.05)$. Furthermore, a significant relationship was observed between commitment to action and self-care behaviors $(\beta=0.37 ; \mathrm{P}<0.01)$.

The relationship between interpersonal influences and self-care behavior was not statistically significant. The pathway from situational influences to self-care behaviors was not significant. However, the pathway between perceived benefit and self-care behaviors was significant $(\beta=0.17 ; \quad P<0.01)$. Moreover, the activity-related affect predicted self-care behaviors

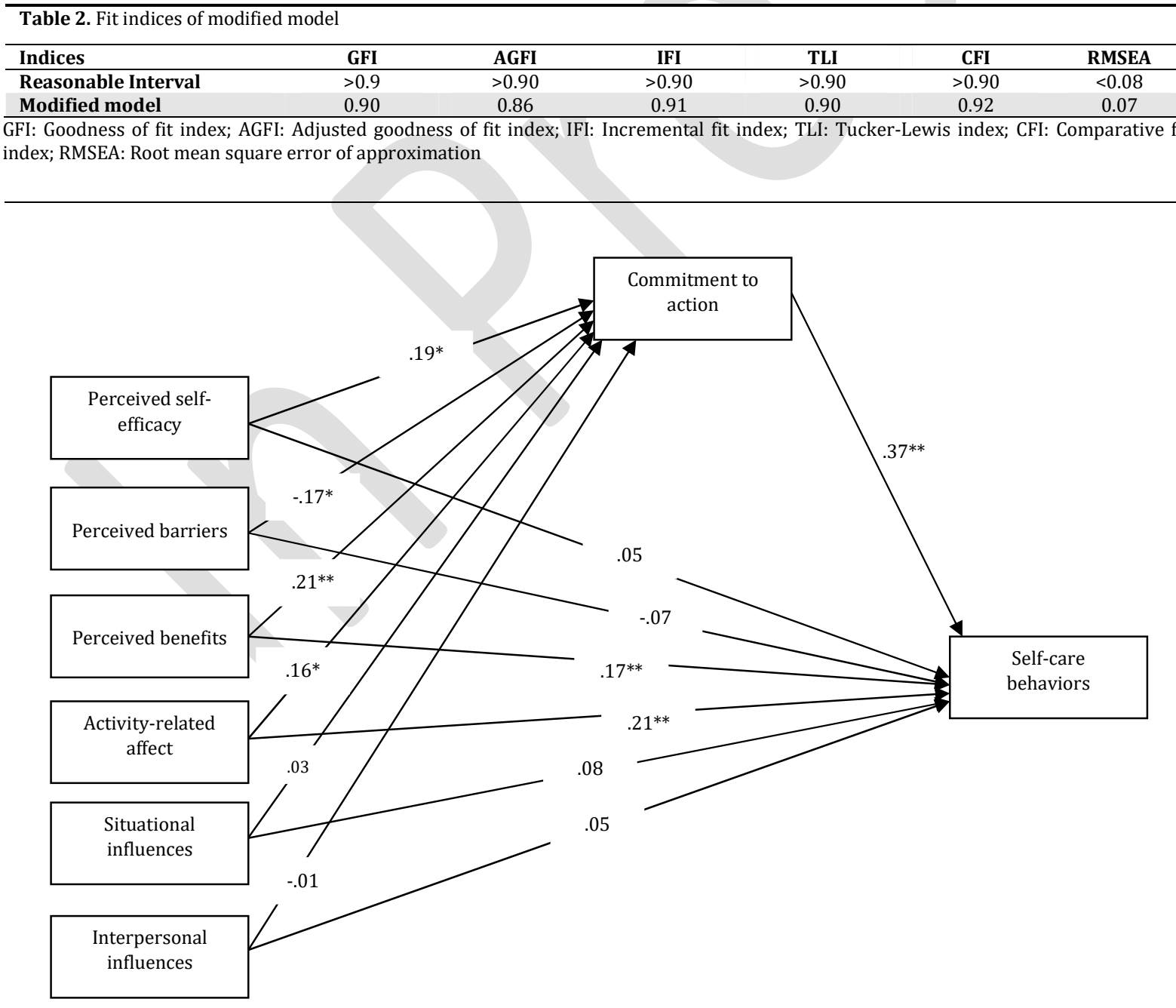

Figure 1. Mediation of commitment to action between predictive variables and self-care behaviors; all parameters are standardized; ${ }^{*} \mathrm{P}<0.05 ;{ }^{* *} \mathrm{P}<0.01$ 


\begin{tabular}{llcccc}
\hline \multicolumn{2}{l}{ Table 3. Bootstrapping results to examine mediating role of commitment to action between independent variables and self-care behaviors } \\
\hline & & & \multicolumn{2}{c}{$\mathbf{9 5 \%}$ Bias-corrected CI } & \multirow{2}{*}{ P-value } \\
\hline Independent variable & Mediator variable & Dependent variable & Lower bound & Upper bound & $0.00^{* *}$ \\
\hline Perceived self-efficacy & Commitment to action & Self-care behaviors & 0.02 & 0.15 & $0.00^{* *}$ \\
Perceived barriers & Commitment to action & Self-care behaviors & -0.02 & -0.14 & $0.00^{* *}$ \\
Perceived benefits & Commitment to action & Self-care behaviors & 0.03 & 0.18 & $0.00^{* *}$ \\
Activity-related affect & Commitment to action & Self-care behaviors & 0.01 & 0.13 & 0.09 \\
Situational influences & Commitment to action & Self-care behaviors & -0.02 & 0.19 & 0.07 \\
\hline Interpersonal influences & Commitment to action & Self-care behaviors & -0.12 & 0.24 & 0.07 \\
\hline Effects are significant when the & uper and lower bounds of bias-corrected 95\% confidence interval do not contain zero. &
\end{tabular}

Effects are significant when the upper and lower bounds of bias-corrected 95\% confidence interval do not contain zero. ** $\mathrm{P}<0.01$

$(\beta=0.21 ; \mathrm{P}<0.01)$.

\subsection{Indirect effects}

To study the indirect effects, the Bootstrap test was used and the results are shown in Table 3. The findings of bootstrapping analysis demonstrated that commitment to action fully mediated the relationship between perceived self-efficacy and self-care behaviors (95\% CI: $0.02-0.15$ ). Since 0 is outside the $0.95 \% \mathrm{CI}$, this path was significant. The indirect path from perceived barriers to self-care behaviors through commitment to action was significant (95\% CI: -0.02 to -0.14$)$. Commitment to action fully mediated the aforementioned relation. Commitment to action had a partial mediating effect on the relationship between perceived benefits and self-care behaviors (95\% CI: 0.03-0.18). Additionally, the indirect path from activity-related affect to self-care behaviors through commitment to action was significant (95\% CI: 0.01-0.13). The indirect effect of situational and interpersonal influences on self-care behaviors via commitment to action was not significant.

\section{Discussion}

The present investigation aimed to study the predictors of self-care behaviors among heart failure patients on the basis of Penders HPM using structural equation modeling. The obtained results of the current study indicated that conceptual HPM is one of the most universal models for the prediction of health-promoting behaviors among the population of patients with cardiovascular diseases in Iran.

The level of self-care behaviors changed by perceived benefits and activity-related affect. These findings are consistent with the results of previous studies (28). In line with the findings of the present study, the results of other studies showed that patients with a better understanding of risk factors and benefits of pursuing health-promoting behaviors have better performance in the implementation of preventive behaviors (29).

The mediation effects of perceived self-efficacy, perceived barriers, perceived benefits, and activityrelated affect through commitment to action to selfcare behaviors were significant. The aforementioned findings are consistent with the results of previous studies $(30,31)$. Since the behaviors are complex phenomena and changing them is a continuous and goal-based process, it is not possible to obtain a logical and purposeful result without relying on a defined and planned commitment (32).

Based on HPM, commitment to action is less likely to lead to desired behaviors. The result of a different study has shown that commitment to action is affected by some variables, such as perceived selfefficacy, perceived barriers, perceived benefits, activity-related affect, situational impacts, and interpersonal influences. Perceived barriers prevent commitment to action, and instead perceived selfefficacy, perceived benefits, and activity-related affect increase commitment to action. Barriers may be imaginary or real in relation to health-promoting behaviors. They are notions of unavailability of sports facilities, responsibility toward family, lack of time, expenses, and taking care of others decreasing health by increasing individuals willingness to avoid and reduce health-promoting behaviors (33). If the perceived barriers outweigh the anticipated benefits, the behavior is less likely to occur (34).

On the other hand, perceived positive or negative emotions occurring before, during, and after behavior based on the stimulus properties of the behavior itself can affect commitment to action. As positive emotions increases, the feeling of efficacy increases. In turn, increased feelings of efficacy can lead to a further positive effect. In addition, perceived selfefficacy can increase control in patients, and then control can affect displaying a healthy behavior. In fact, it can be said that once a patient s ability to control symptoms is high, the beginning of self-care behaviors also promote.

According to the results of the present study, individuals appropriately evaluating their health status considered themselves capable of performing health-promoting behaviors and felt more selfefficacy. There have been multiple studies indicating that self-efficacy has been identified as a determinant of health-promoting behaviors (35-37). According to social cognitive theory, individual judgment based on ability will affect behaviors, such as success in displaying self-care behaviors. Individuals with high self-control believed that they are able to effectively affect their life events and expect more success, compared to those with low self-control. These 
individuals do not drown in their skepticism, look at difficult tasks as challenges not as threats, and are usually looking for challenges and responding to them (38-40). Therefore, according to the role of selfcontrol in the modification of self-care behaviors and promotion of commitment to action, it is necessary to consider the improvement of this ability in patients with heart failure in designing educational and therapeutic programs.

\subsection{Limitations}

In the present study, there were some limitations. Firstly, convenience sampling was utilized in this study leading to the restriction on the generalizability of the results. Secondly, the educational levels of the participants were predominantly under diploma and diploma, and patients with bachelor, master, and higher education levels were underrepresented. This demographic characteristic also restricted the generalizability of the findings of this study. Therefore, the results of the present study should be interpreted with caution due to non-probability sampling and demographic characteristics of the study population. Finally, all of the determinants that may affect self-care behaviors, especially education, drug use, and family support, were not noticed in the current study. Consequently, the results of this study were limited in representing the conclusive determinants of self-care behaviors in heart failure patients.

\section{Conclusion}

According to the obtained results of the present study, commitment to action was affected by predictive variables, such as perceived self-efficacy, perceived barriers, and perceived benefits. Perceived barriers can prevent commitment to action and selfcare behavior, and inversely activity-related affect and self-efficacy can promote commitment to action and self-care behavior in patients with heart failure. Therefore, the interventions should take the role of these predictive variables and commitment to action into account among these patients. Specialists can plan educational programs based on Pender's HPM in which the variables affecting commitment to action and self-care behavior are considered.

According to the results of the current study, patients and their relatives can increase their awareness of disease symptoms, perceived benefits, and perceived barriers, thereby increasing their health literacy. The establishment of appropriate health programs and provision of simple and comprehensible educational materials are the strategies helping patients with low levels of health literacy and improving their knowledge.

\section{Acknowledgments}

The authors would like to express their gratitude to all the health personnel and patients who participated in this study.

\section{Footnotes}

Authors' Contribution: Rasoul Heshmati concepttualized the study and analyzed and interpreted the data. Mahasti Alizadeh supervised the study. Solmaz Javadpour collected the data. Rasoul Heshmati and Nilufar Alizada contributed to writing the manuscript, especially the introduction and discussion. All the authors contributed to preparing the final version of the manuscript.

Conflict of Interests: The authors declare that they have no competing interests.

Ethical Approval: The present research project was approved by the Research Ethics Committee of University of Tabriz, Iran (IR.TABRIZU.REC.1399.051).

Funding/Support: This study was supported by the Social Determinants of Health Research Center, Health Management and Safety Promotion Research Institute, Tabriz University of Medical Sciences.

Informed consent: Written informed consent was obtained from all the patients who participated in the present study.

\section{References}

1. Hatmi ZN, Tahvildari S, Motlag AG, Kashani AS. Prevalence of coronary artery disease risk factors in Iran: A population based survey. BMC Cardiovasc Disord. 2007;7:32. doi: 10.1186/1471-2261-7-32. [PubMed: 17971195].

2. Goodie, Jeffrey L., Kevin Wilfong, and Phillip Kroke. "The influence of health behaviors upon the association between stress and depression and cardiovascular disease. Cardiovascular Implications of Stress and Depression. Academic Press, 2020. P:225-254.

3. Bui AL, Horwich TB, Fonarow GC. Epidemiology and risk profile of heart failure. Nat Rev Cardiol. 2011;8(1):30-41. doi: 10.1038/nrcardio.2010.165. [PubMed: 21060326].

4. Norton C, Georgiopoulou VV, Kalogeropoulos AP, Butler J. Epidemiology and cost of advanced heart failure. Prog Cardiovasc Dis. 2011;54(2):78-85. doi: 10.1016/j. pcad.2011. 04.002. [PubMed: 21875507].

5. Miller LW. Heart failure: who we treat versus who we study. Cardiol Clin. 2008;26(1):113-25. doi: 10.1016/j. ccl.2007.10.005. [PubMed: 18312911].

6. Benjamin EJ, Blaha MJ, Chiuve SE, Cushman M, Das SR, Deo R, et al. Heart disease and stroke statistics-2017 update: a report from the American Heart Association. Circulation. 2017;135(10):e146-e603. doi: 10.1161/CIR.0000000000000 485. [PubMed: 28122885].

7. Goldberg RJ, Ciampa J, Lessard D, Meyer TE, Spencer FA. Longterm survival after heart failure: a contemporary populationbased perspective. Arch Intern Med. 2007;167(5):490-6. doi: 10.1001/archinte.167.5.490. [PubMed: 17353497].

8. Johansson P, Brostron A, Dahlsron U, Alehagen U. Global perceived health and health-related quality of life in elderly primary care patients with symptoms of heart failure. Europ J Cardiov Nurs. 2008;7(4): 96-76. doi: 10.1016/j.ejcnurse.2007. 12.002 .

9. Macabasco-O'Connell A, Crawford MH, Stotts N, Stewart A, Froelicher ES. Self-care behaviors in indigent patients with heart failure. Eur J Cardiovasc Nurs 2008;23(3):223-30. doi: 10.1097/01.JCN.0000317427.21716.5f. [PubMed: 18437064].

10. Siabani S, Driscoll T, Davidson PM, Najafi F, Jenkins MC, Leeder 
SR. Self-care and Its Predictors in Patients With Chronic Heart Failure in Western Iran. J Cardiovasc Nurs. 2016;31(1):22-30. doi: 10.1097/JCN.0000000000000211. [PubMed: 25419944].

11. Karasu F, Aylaz R. Evaluation of meaning of life and self-care agency in nursing care given to chronic obstructive pulmonary patients according to health promotion model. Appl Nurs Res. 2020;51:151208. doi: 10.1016/j.apnr.2019.151208. [PubMed: 31676298].

12. Jaarsma T, Halfen $R$, sSenten $M$, Huijer AH, Dracup $K$ Developing a supportive educative program for patient with advanced HF within orems general theory nursing. Nurs Sci Quarter. 1998;2:79-85. doi: 10.1177/089431849801100210.

13. Gary R. Self-care practices in woman with diastolic heart failure. Heart Lung. 2006;35(1):9-19. doi: 10.1016/j.hrtlng. 2005.04.005.

14. Sakhayi S. The study of applying rate of life style healthy promotion method in medical personal. Zahedan J Res Med Sci. 2005;7(2):721-4.

15. Pender NJ, Murdaugh CL, Parsons MA. Health Promotion in Nursing Practice.4th ed. Upper Saddle River NJ: Prentic Hall; 2002.P.57-64.

16. Wai Sze Lo S, Ying Chair S, Kam Lee F. Factors associated with health-promoting behavior of people with or at high risk of metabolic syndrome: Based on the health belief model. App nurs Res. 2015;28:197-201. doi: 10.1016/j.apnr.2014.11.001. [PubMed: 25540911].

17. Weston NM. Identifying perceptions of health promotion barriers and benefits in individuals at risk for coronary heart disease. Montana;2008.

18. Thanavaro J. Barriers to coronary heart disease risk modification in women without prior history of coronary heart disease. J Am Aca Nurs Pract. 2005;17:487-93. doi: 10.1111/j.1745-7599.2005.00080.x. [PubMed: 16248882].

19. Mosca L, Mochari H, Christian A, Berra K, Taubert K, Mills T National study of women's awareness, preventive action, and barriers to cardiovascular health. Circulation. 2006;113(4) 525-34. doi: 10.1161/CIRCULATIONAHA.105.588103. [PubMed: 16449732]

20. Aljasem LI, Peyrot M, Wissow L, Rubin RR. The impact of barriers and self efficacy on self-care behaviors in type 2 diabetes. Diabetes Edu. 2001;27(3):393-404. doi: 10.1177/014572170102700309. [PubMed: 11912800].

21. Salehzadeh A, Rahmatpour P. Self-care behaviors and related factors in patients with heart failure reffering to medical \& educational center of heart in Rasht. J Holc Nurs And Midwif 2013;23(1):22-9.

22. Kamrani AA, Foroughan M, Taraghi Z, Yazdani J, Kaldi AR, Ghanei N,et al. Self care behaviors among elderly with chronic heart failure and related factors. Pak J Biol Sci. 2014; 17(11):1161-9. doi: 10.3923/pjbs.2014.1161.1169. [PubMed: 26027161].

23. Smith MS, Wallston KA, Smith CA. The development and validation of the Perceived Health Competence Scale. Health Educ Res. 1995;10(1):51-64. doi: 10.1093/her/10.1.51. [PubMed: 10150421]

24. Becker H, Stuifbergen A. What makes it so hard? Barriers to health promotion experienced by people with multiple sclerosis and polio. Fam Community Health. 2004;27(1):7585. doi: 10.1097/00003727-200401000-00008. [PubMed: $14724504]$.

25. Mohammadian H, Eftekhar Ardebili H, Rahimi Foroushani A Taghdisi MH, Shojaei Zadeh D. Evaluation of pender health promotion model in predicting living quality of adolescent girls. J Faculty Health Health Res Inst. 2011;8(4):1-13.

26. Watson D, Clark LA, Tellegen A. Development and validation of brief measures of positive and negative affect: the PANAS scales. J Pers Soc Psychol.1988;54(6):1063-70. doi: 10.1037//0022-3514.54.6.1063. [PubMed: 3397865].

27. Canty-Mitchell J, Zimet GD. Psychometric properties of the mul-tidimensional Scale of perceived social support in urban adolescents. Am J Community Psychol.2000;28(3):391-400. doi: 10.1023/A:1005109522457. [PubMed: 10945123].

28. Bahmanpour K, Nouri R, Nadrian H, Salehi B. Determinants of oral health behavior among high school students in Marivan County, Iran based on the Pender's Health Promotion Model. Sch Pub Health Ins Pub Health Res. 2011;9(2):93-106.

29. Zeighami Mohammadi S, Fahidy F. Perception of patients with cardiovascular disease about barriers and benefits of health promotion behaviors. J Cardiovasc Nurs. 2012;1(2):16-23.

30. Alizadeh Charandabi, SM, Mirghafourvand M, Tavananezhad N, Karkhaneh M. Health promoting lifestyles and self-efficacy in male adolescents in Sanandaj. I mazandaran univ med sci. 2014;23(109):152-62.

31. Oestrich IH, Austin SF, Lykke J, Tarrier N. The feasibility of a cognitive behavioural intervention for low self-esteem within a dual diagnosis inpatient population. Behav Cogn Psychother. 2007;35(4):403-8. doi: 10.1017/S1352465807003633.

32. Forrest JL, Overman P. Keeping current: a commitment to patient care excellence through evidence based practice. J Dent Hyg. 2013;87(1): 33-40. [PubMed: 24046340]

33. Mohamadian H, Eftekhar Ardebili H, Rahimi Foroushani A, Taghdisi MH, Shojaiezade D . Evaluation of Pender's health promotion model for predicting adolescent girls' quality of life. Sci J Sch Public Health Ins Public Health Res. 2011;8(4):1-13.

34. Koerber A, Peters KE, Kaste LM, Lopez E, Noorullah K, Torres I, et al. The views of dentists, nurses and nutritionists on the association between diabetes and periodontal disease: a qualitative study in a Latino community. J Public Health Dent. 2006;66(3):212-5. doi: 10.1111/j.1752-7325.2006.tb02583.x. [PubMed: 16913250].

35. Christensen AJ, Wiebe JS, Benotsch EG, Lawton WJ. Perceived health competence, health locus of control, and patient adherence in renal dialysis. Cognit Ther Res. 1996;20(4):411-21.

36. Bayrami M, Heshmati R, Ghotbi M, Ghoradel JA, Hojatipor H, Moslemifar M. Relationship between personality dimensions and hopelessness: a study on college students. Procedia-Soci Behav Sci. 2012;46:848-52. doi: 10.1016/j.sbspro.2012.05.211.

37. Shaker A, Heshmati R, Rahimi MP. Investigation of marital adjustment in people with secure, preoccupied, dismissing and fearful attachment styles. Procedia-Soci Behav Sci. 2010; 5:1823-6. doi: 10.1016/j.sbspro.2010.07.371.

38. Kadivar M, Kahrazei F, Nikmanesh Z. The effectiveness of interventions based on positive psychotherapy on improving coping styles, relapse prevention in individual diagnosed with substance use disorder. Positive Psychol . 2016;1(4): 41-54.

39. Heshmati R, Ghorbani F. The effect of Mindfulness-Based Stress Reduction (MBSR) program on physical functioning and health related quality of life (HRQOL) in people with coronary artery disease (CAD). Iran J Cardiovascul Nurs. 2016;5(3):16-25.

40. Heshmati R. Structural relationships among functional status, health beliefs and bmi in patients with cad: the mediator role of cardiac self-efficacy. J Health Care. 2016;18(3):191-206. 